\title{
A WREATH PRODUCT APPROACH TO CLASSICAL SUBGROUP THEOREMS
}

\author{
by Luis RiBES and Benjamin STEINBERG*)
}

ABSTRACT. We provide elementary proofs of the Nielsen-Schreier Theorem and the Kurosh Subgroup Theorem via wreath products. Our proofs are diagrammatic in nature and work simultaneously in the abstract and profinite categories. A new proof that open subgroups of quasifree profinite groups are quasifree is also given.

\section{INTRODUCTION}

The purpose of this paper is to provide a conceptual framework for simple algebraic proofs, in both their abstract and profinite versions, of the classical subgroup theorems from combinatorial group theory: the Nielsen-Schreier Theorem and the Kurosh Theorem. Our proof of the Nielsen-Schreier Theorem, for instance, could very easily be presented in a first course introducing free groups. The fundamental idea is to exploit the functoriality of the wreath product in order to reduce these theorems to diagram chasing. By removing as much as possible the combinatorics on words, we are able to present proofs that also work in the profinite category. Traditionally, the subgroup theorems for profinite groups are obtained via a reduction to the abstract case; here we prove the abstract and profinite theorems simultaneously.

In addition to proving the classical subgroup theorems, we also give a very simple and natural proof of a result of the first author, Stevenson and Zalesskii [17] on open subgroups of quasifree profinite groups.

The origins of our approach via wreath products lie in two sources: profinite group theory and profinite semigroup theory. The genesis of the wreath product technique for subgroup theorems is [4], where Cossey, Kegel and Kovács used wreath products to prove that closed subgroups of projective profinite groups are again projective. The usual proofs of this result rely on

*) The authors gratefully acknowledge the support of NSERC. 
the Nielsen-Schreier Theorem for abstract free groups or on cohomological techniques, see [18, Theorem 7.7.4] for example. The wreath product approach was further developed by Haran to study closed subgroups of free products of profinite groups [8]. Ershov [6] seems to be the first to have attempted to use wreath products to deal with subgroup theorems for discrete groups. In particular, he gives a proof of the Kurosh Theorem using Haran's notion of a projective family. However, his proof is not conceptually appealing since it follows this route of projective families, and moreover it relies on the NielsenSchreier Theorem, which normally should be deducible as a special case of the Kurosh Theorem.

The same wreath product techniques arose independently in the work of semigroup theorists investigating the structure of free profinite monoids. Wreath products were first introduced into semigroup theory by Schützenberger [19] and came to play a major role in the subject with the advent of the Krohn-Rhodes Theorem [12], which definitively established the wreath product as the principal instrument for decomposing semigroups into simpler parts; see Eilenberg's book [5] or [16] for details. There is no Nielsen-Schreier Theorem for free monoids; also cohomological techniques do not work well for semigroups because the Eckmann-Shapiro Lemma fails in this context. Semigroup theorists were then naturally led to the wreath product to prove structural results about free profinite monoids. Margolis, Sapir and Weil [14] first exploited this technique in order to show that those finitely generated clopen submonoids of a free profinite monoid that have any chance to be free are indeed free; this was extended to the non-finitely generated case by Almeida and the second author [1]. Rhodes and the second author rediscovered the proof from [4] that closed subgroups of projective profinite groups are projective and used an analogous argument to establish that closed subgroups of free profinite monoids are projective profinite groups [15]; see also [20] where a similar idea was used.

We soon came to realize that the theorems for abstract groups should also be amenable to these techniques, leading to the current paper. The paper is organized as follows. The first section sets up our notation for wreath products and establishes the basic functorial properties of this construction. Next we turn to the Nielsen-Schreier Theorem, which is proved for abstract groups and then adapted to profinite groups. The Nielsen-Schreier Theorem is followed up by the Kurosh Theorem, which is the most technical part of the paper. The paper closes with a proof that open subgroups of quasifree profinite groups are again quasifree. The aim of this paper is to be a self-contained and elementary exposition, so many well-known results are included. 


\section{NOTATION AND CONVENTIONS}

If $K$ and $L$ are groups, $K \leq L$ indicates that $K$ is a subgroup of $L$. Composition of maps in this paper is always assumed to be right-to-left, except when dealing with permutations in a symmetric group $S_{\Sigma}$, which we multiply left-to-right. If $x, y \in L$, we define $x^{y}=y^{-1} x y$ and $K^{y}=y^{-1} K y$. The inner automorphism $\operatorname{inn}_{y}$ of $L$ determined by $y$ is the automorphism $x \mapsto y x y^{-1}$ $(x \in L)$.

\subsection{THE SEMIDIRECT PRODUCT}

Recall that a group $G$ is said to act on a group $R$ on the left, if there exists a homomorphism $\alpha: G \rightarrow \operatorname{Aut}(R)$ denoted by $g \mapsto \alpha_{g}(g \in G)$.

Equivalently, $G$ acts on $R$ on the left if there is a function $G \times R \rightarrow R$ denoted by $(x, r) \mapsto{ }^{x} r$, such that

(a) ${ }^{1} r=r, \forall r \in R$,

(b) ${ }^{x y} r={ }^{x}\left({ }^{y} r\right), \forall r \in R, x, y \in G$,

(c) ${ }^{x}\left(r_{1} r_{2}\right)={ }^{x} r_{1}{ }^{x} r_{2}, \forall r_{1}, r_{2} \in R, x \in G$.

Indeed, just define ${ }^{x} r=\alpha_{x}(r)$.

Given such an action, define the corresponding semidirect product $R \rtimes G$ to be the group with underlying set $R \times G$ and multiplication given by

$$
(r, x)\left(r_{1}, x_{1}\right)=\left(r\left({ }^{x} r_{1}\right), x x_{1}\right) \quad\left(r, r_{1} \in R, x, x_{1} \in G\right) .
$$

One checks that indeed this multiplication makes $R \rtimes G$ into a group with identity element $(1,1)$. Note that

$$
(r, x)^{-1}=\left({ }^{x^{-1}}\left(r^{-1}\right), x^{-1}\right), \quad(1, x)(r, 1)(1, x)^{-1}=\left({ }^{x} r, 1\right) .
$$

Moreover, the maps

$$
R \rightarrow R \rtimes G \quad r \mapsto(r, 1) \quad(r \in R) \quad \text { and } \quad G \rightarrow R \rtimes G \quad x \mapsto(1, x) \quad(x \in G)
$$

are injective homomorphisms. If we identify $R$ and $G$ with their images under these injections, we have $R \rtimes G=R G$, with $R \cap G=1$ and $R \triangleleft R \rtimes G$. When using this identification we sometimes write the elements of $R \rtimes G=R G$ as $r \cdot x(r \in R, x \in G)$. Throughout the paper we use the notation $(r, x)$ or $r \cdot x$ for an element of $R \rtimes G$, according to convenience.

\subsection{PERMUTATIONAL WREATH PRODUCTS}

Fix a set $\Sigma$. Given a group $A$, define $A^{\Sigma}$ to be the group of all functions $f: \Sigma \rightarrow A$. We write the argument of such a function $f$ on its right; thus the 
operation on $A^{\Sigma}$ is given by

$$
(f g)(s)=f(s) g(s) \quad\left(f, g \in A^{\Sigma}, s \in \Sigma\right) .
$$

We denote by $\delta: A \rightarrow A^{\Sigma}$ the diagonal homomorphism: it assigns to $a \in A$ the constant function $\delta_{a} \in A^{\Sigma}$ defined by $\delta_{a}(s)=a$, for all $s \in \Sigma$. The image of $\delta$ is denoted $\delta_{A}$.

Assume that a group $G$ acts on $\Sigma$ on the right. Define the permutational wreath product $A<G$ (with respect to the $G$-set $\Sigma$ ) to be the semidirect product

$$
A \prec G=A^{\Sigma} \rtimes G,
$$

where the action of $G$ on $A^{\Sigma}$ is defined by

$$
g_{f}(s)=f(s g) \quad\left(g \in G, f \in A^{\Sigma}, s \in \Sigma\right) .
$$

The usage of left exponentiation follows Eilenberg [5]. Observe that $G$ centralizes $\delta_{A}$ in $A \succ G$, so that $\left\langle\delta_{A}, G\right\rangle=\delta_{A} \times G$.

2.2.1 ELEMENTARY PROPERTIES. Several fundamental properties of the wreath product are recorded in the following proposition.

PROPOSITION 2.2.1. (a) If $B \leq A$ are groups, and $H \leq G$, then

$$
B \nmid H=B^{\Sigma} \rtimes H \leq A \prec G=A^{\Sigma} \rtimes G .
$$

(b) Functoriality on $A:(-) 2 G$ is a functor, i.e., for each homomorphism $\alpha: A \rightarrow B$, there is a homomorphism

$$
\alpha \prec G: A \prec G=A^{\Sigma} \rtimes G \rightarrow B \prec G=B^{\Sigma} \rtimes G
$$

given by $(f, g) \mapsto(\alpha f, g)$, where $f \in A^{\Sigma}$ and $g \in G$, so that

(b1) $\operatorname{id}_{A} \succ G=\operatorname{id}_{A \nmid G}$, and

(b2) if $A \stackrel{\alpha}{\rightarrow} B \stackrel{\beta}{\rightarrow} C$ are group homomorphisms, then

$$
\beta \alpha \curlyvee G=(\beta \curlyvee G)(\alpha \succ G) \text {. }
$$

(c) Furthermore, $\alpha 2 G$ is an epimorphism (respectively, a monomorphism) if and only if $\alpha$ is an epimorphism (respectively, a monomorphism). 


\subsection{THE STANDARD EMBEDDING}

Let $H$ be a subgroup of a group $G$. Let $\Sigma=H \backslash G$ be the set of all right cosets of $H$ in $G$. Denote by $\rho: G \rightarrow S_{\Sigma}$ the associated permutation representation of $G$ in $S_{\Sigma}$, i.e., $\rho$ is the homomorphism defined by $\rho(g)=\bar{g}$ $(g \in G)$, where $\bar{g}: \Sigma \rightarrow \Sigma$ is the permutation $H x \mapsto H x g \quad(x \in G)$. Note that

$$
\operatorname{ker}(\rho)=\bigcap_{x \in G} x H x^{-1}=H_{G}
$$

the core of $H$ in $G$.

Fix a right transversal $T$ of $H$ in $G$, i.e., a complete set of representatives of the right cosets $H x \quad(x \in G)$. We denote the representative of $H x$ in $T$ by either $t_{H x}$ or $\bar{x}$, as convenient. Define $s_{T} \in G^{\Sigma}$ as the map that assigns to each right coset of $H$ in $G$ its representative in $T$ :

$$
s_{T}(H x)=t_{H x}=\bar{x} \in T \quad(x \in G) .
$$

Consider the monomorphism of groups $\widetilde{\varphi}: G \rightarrow G 々 \rho(G)$ given by the composition of homomorphisms

$$
G \stackrel{\delta \times \rho}{\longrightarrow} \delta_{G} \times \rho(G) \longleftrightarrow G\left(\rho(G) \stackrel{\operatorname{inn}_{S_{T}}}{\longrightarrow} G\right\urcorner \rho(G) .
$$

Explicitly, if $g \in G$, then

$$
\widetilde{\varphi}(g)=s_{T}\left(\delta_{g} \cdot \rho(g)\right) s_{T}^{-1}=f_{g} \cdot \rho(g),
$$

where $f_{g} \in G^{\Sigma}$ is defined by $f_{g}=s_{T} \delta_{g}{ }^{\rho(g)}\left(s_{T}^{-1}\right)$, i.e.,

$$
f_{g}(H x)=t_{H x} g t_{H x g}^{-1} \quad(x \in G) .
$$

We remark that $\widetilde{\varphi}(G) \leq H \imath \rho(G)$, because $f_{g}(H x)=t_{H x} g t_{H x g}^{-1} \in H(x \in G)$. Therefore, we have proved

THEOREM 2.3.1 (Embedding Theorem). Let $H \leq G$ be groups.

(a) There is an injective homomorphism $\varphi: G \rightarrow H>\rho(G)$ defined by

$$
\varphi(g)=f_{g} \cdot \rho(g),
$$

where $f_{g}: \Sigma=H \backslash G \rightarrow H$ is given by $f_{g}(H x)=t_{H x} g t_{H x g}^{-1}(g, x \in G)$.

(b) $\left.\varphi\right|_{H}(H) \leq H^{\Sigma} \rtimes \rho(H)=H \imath \rho(H)$.

We record the following facts for future use; they follow by routine computation in the wreath product. 
LEMMA 2.3.2. Let $A$ be a group and $\psi: G \rightarrow A \curlywedge \rho(G)$ be a homomorphism such that

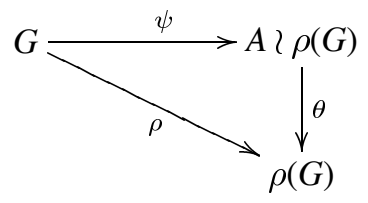

commutes, where $\theta$ is the projection. Put $\psi(g)=\left(\tilde{f}_{g}, \rho(g)\right)(g \in G)$. Then the following hold:
(a) $\widetilde{f}_{g_{1} g_{2} \cdots g_{n}}=\widetilde{f}_{g_{1}} \rho\left(g_{1}\right) \widetilde{f}_{g_{2}} \ldots \rho\left(g_{1} \cdots g_{n-1}\right) \widetilde{f}_{g_{n}} \quad$ for $g_{1}, \ldots, g_{n} \in G$;
(b) $\widetilde{f}_{g^{-1}}=\left(\rho\left(g^{-1}\right) \widetilde{f}_{g}\right)^{-1}={ }^{\rho\left(g^{-1}\right)}\left(\widetilde{f}_{g}^{-1}\right)$ for $g \in G$.

REMARK 2.3.3. If $H \triangleleft G$, then $\Sigma$ has the structure of a group that we denote $K$. Identifying $K$ with its canonical image in $S_{\Sigma}=S_{K}$, we have $K=\rho(G)$, so that $\varphi: G \hookrightarrow H \imath K$. This is the so called Kaluznin-Krasner Theorem: every extension of a group $H$ by a group $K$ can be embedded in $H$ Z $K$ [11]. The standard embedding is very closely related to the monomial map [7, Chap.14] and the theory of induced representations; see [21] for a detailed discussion.

From now on we shall use the notation $T=\left\{t_{i} \mid i \in I\right\}$ (if $T$ is finite, we write $T=\left\{t_{1}, \ldots, t_{k}\right\}$ ), and we shall assume that there is a symbol $1 \in I$ such that $t_{1}=1$ is the representative of the coset $H$, i.e., $t_{H}=t_{1}=1$. Fix $i \in I$. Then the action of $H^{t_{i}}=t_{i}^{-1} H t_{i}$ on $\Sigma=H \backslash G$ fixes the element $H t_{i} \in \Sigma$. Hence if $A$ is a group and $f \in A^{\Sigma}$, one has ${ }^{\rho(x)} f\left(H t_{i}\right)=f\left(H t_{i}\right)$, for all $x \in H^{t_{i}}$. Therefore, the copy

$$
\left\{f\left(H t_{i}\right) \mid f \in A^{\Sigma}\right\} \cong A
$$

of the group $A$ corresponding to the $H t_{i} \in \Sigma$ component of the direct product $A^{\Sigma}$ centralizes $\rho\left(H^{t_{i}}\right)$ in $A \prec \rho\left(H^{t_{i}}\right)$. Thus

$$
A \prec \rho\left(H^{t_{i}}\right)=A^{\Sigma} \rtimes \rho\left(H^{t_{i}}\right)=A \times\left(A^{\Sigma-\left\{H t_{i}\right\}} \rtimes \rho\left(H^{t_{i}}\right)\right) .
$$

We denote by $\pi_{A, i}: A \nmid \rho\left(H^{t_{i}}\right) \rightarrow A$ the corresponding projection:

$$
\pi_{A, i}(f \cdot \rho(x))=f\left(H t_{i}\right) \quad\left(x \in H^{t_{i}}, f \in A^{\Sigma}\right) .
$$

The case $i=1$ will be used so often, that it is convenient to set $\pi_{A}=\pi_{A, 1}$. Part (b) of the following lemma expresses the naturality of $\pi_{A, i}$. 
LEMMA 2.3.4. We continue with the above setting. Let $i \in I$.

(a) There is a commutative diagram

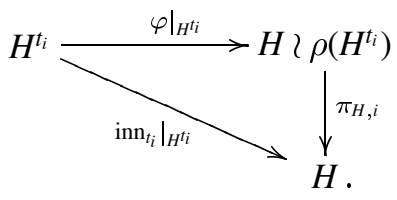

In particular, for $i=1,\left.\pi_{H} \varphi\right|_{H}=\left.\pi_{H, 1} \varphi\right|_{H}=\mathrm{id}_{H}$.

(b) If $\alpha: A \rightarrow B$ is a homomorphism of groups, then the diagram

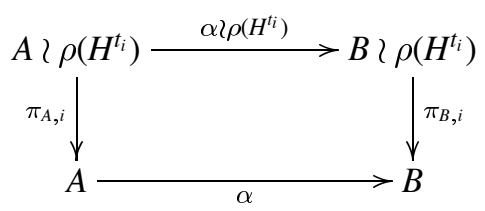

commutes.

(c) One has $\bigcap_{i \in I}\left(A<\rho\left(H^{t_{i}}\right)\right)=A 々 \rho\left(H_{G}\right)=A^{\Sigma}$, where $H_{G}$ is the core of $H$. The restriction $\left.\left(\pi_{A, i}\right)\right|_{A^{\Sigma}}: A^{\Sigma} \longrightarrow A$ is the usual direct product projection.

Proof. To prove (a) observe that, for $r \in H^{t_{i}}$, one has

$$
\pi_{H, i} \varphi(r)=f_{r}\left(H t_{i}\right)=t_{H t_{i}} r t_{H t_{i} r}^{-1}=t_{i} r t_{i}^{-1}
$$

since $H^{t_{i}}$ stabilizes $H t_{i}$. The proof of (b) follows directly from the definitions of $\pi_{A, i}, \pi_{B, i}$, and $\alpha 2 \rho\left(H^{t_{i}}\right)$. Part (c) is clear, as $\bigcap_{i \in I} H^{t_{i}}=H_{G}=\operatorname{ker} \rho$.

\subsection{THE EMBEDDING THEOREM FOR PROFINITE GROUPS}

By a variety of finite groups we mean a nonempty class $\mathcal{C}$ of finite groups closed under taking subgroups, finite direct products and homomorphic images. In this paper, we assume in addition that the variety $\mathcal{C}$ is closed under extensions of groups (we say then that $\mathcal{C}$ is an extension closed variety of finite groups). A pro- $\bigodot$ group is a profinite group whose continuous finite quotients are in $\mathcal{C}$, i.e., an inverse limit of groups in $\mathcal{C}$. Suppose now that $G$ is a pro- $\bigodot$ group and $H$ is an open subgroup of $G$ (cf. [18] for basic properties of profinite groups). Let $\Sigma=H \backslash G$; then $\Sigma$ is finite, and the quotient topology on $\Sigma$ is discrete. Let $\rho: G \rightarrow S_{\Sigma}$ be as before; since $H_{G}=\operatorname{ker} \rho$ is open in $G$, the homomorphism $\rho$ is continuous. If $A$ is any pro- $\mathcal{C}$ group, then $A^{\Sigma}$ is a pro- $\mathcal{C}$ group and the left action $\rho(G) \times A^{\Sigma} \rightarrow A^{\Sigma}$, as defined above, is continuous since $\Sigma$ and $\rho(G)$ are finite and the action just permutes the 
coordinates. Thus the wreath product $A \curlywedge \rho(G)=A^{\Sigma} \rtimes \rho(G)$ is a pro- $\bigodot$ group (here we use that $\mathcal{C}$ is extension closed). Moreover, if $T$ is a transversal for $\Sigma$, then it follows immediately from the definition that the standard embedding $\varphi: G \rightarrow H 2 \rho(G)$ is continuous.

\section{THE NIELSEN-SCHREIER THEOREM}

We present an elementary proof of the Nielsen-Schreier Theorem, stating that subgroups of free groups are free, using wreath products. Our proof is algebraic in nature, rather than combinatorial, and proceeds by direct verification of the universal property. Let $F$ be a free group on $X$ and $H$ a subgroup. Elements of $F$ can be viewed as reduced words over $X \cup X^{-1}$ [13].

\subsection{SCHREIER TRANSVERSALS}

A Schreier transversal for $H \leq F$ is a right transversal $T$ of $H$ in $F$ that is closed under taking prefixes (and in particular contains the empty word): if $y_{1} \cdots y_{i} \cdots y_{n} \in T$ with $y_{1}, \ldots, y_{n} \in X \cup X^{-1}$ in reduced form, then $y_{1} \cdots y_{i} \in T$, for all $i=0, \ldots, n-1$. The existence of Schreier transversals is a standard exercise in Zorn's Lemma.

Lemma 3.1.1. There exists a Schreier transversal $T$ of $H$ in $F$.

Proof. Consider the collection $\mathcal{P}$ of all prefix-closed sets of reduced words in $X \cup X^{-1}$ that intersect each right coset of $H$ in at most one element, and order $\mathcal{P}$ by inclusion. Then $\{1\} \in \mathcal{P}$, so it is non-empty. It is also clear that the union of a chain of elements from $\mathcal{P}$ is again in $\mathcal{P}$, so $\mathcal{P}$ has a maximal element $T$ by Zorn's Lemma. We need to show that each right coset of $H$ has a representative in $T$. Suppose this is not the case and choose a minimum length word $w$ so that $H w \cap T=\varnothing$. Since $1 \in T$, it follows that $w \neq 1$ and hence $w=u x$ in reduced form, where $x \in X \cup X^{-1}$. By assumption on $w$, we have $H u=H t$ for some $t \in T$. If $t x$ is reduced as written, then $T \bullet\{t x\} \in \mathcal{P}$, contradicting the maximality of $T$. If $t x$ is not reduced as written, then $t x \in T$ by closure of $T$ under prefixes and $H w=H t x$, contradicting the choice of $w$. This completes the proof that $T$ is a transversal.

\subsection{THE NiELSEN-SCHREIER THEOREM}

We now proceed with our proof that subgroups of free groups are free via wreath products. 
THEOREM 3.2.1 (Nielsen-Schreier). Subgroups of free groups are free. More precisely, let $F$ be a free group on $X$ and let $H$ be a subgroup. Let $T$ be a Schreier transversal for $H$ and

$$
B=\left\{t x(\overline{t x})^{-1} \mid(t, x) \in T \times X, t x(\overline{t x})^{-1} \neq 1\right\} .
$$

Then $H$ is freely generated by $B$.

Proof. Our goal is to show that any map $\alpha: B \rightarrow G$ with $G$ a group extends uniquely to a homomorphism $\gamma: H \rightarrow G$. First define an extension $\alpha: B \cup\{1\} \rightarrow G$ by $\alpha(1)=1$. Denote by $\Sigma$ the set $H \backslash F$ of right cosets of $H$ in $F$ and let $\rho: F \rightarrow S_{\Sigma}$ be the associated permutation representation of $F$.

To motivate our construction of the extension, we start with a proof of uniqueness. So let $\gamma: H \rightarrow G$ be any homomorphism extending $\alpha$. Consider the standard wreath product embedding $\varphi: F \rightarrow H \imath \rho(F)$ of Theorem 2.3.1. The functoriality of the wreath product and Lemma 2.3.4 yield the commutative diagram

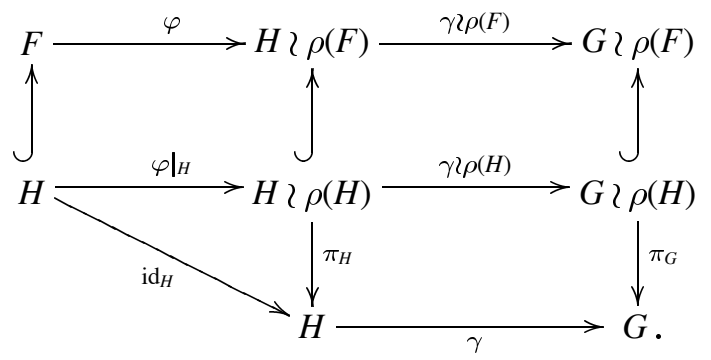

Hence $\gamma$ is uniquely determined by $(\gamma<\rho(F)) \varphi$, which is in turn determined by its values on $X$. But if $x \in X$, then $(\gamma<\rho(F)) \varphi(x)=\left(\gamma f_{x}, \rho(x)\right)$. Now recall that $f_{x}(H w)=t_{H w} x t_{H w x}^{-1} \in B \cup\{1\}$ and hence $\gamma f_{x}=\alpha f_{x}$. Thus the unique possible extension of $\alpha$ to a homomorphism is given by $\pi_{G}\left(\left.\tau\right|_{H}\right)$, where $\tau: F \rightarrow G \backslash \rho(F)$ is the homomorphism defined on $X$ by $\tau(x)=\left(\alpha f_{x}, \rho(x)\right)$. Let us show that $\pi_{G}\left(\left.\tau\right|_{H}\right)$ extends $\alpha$.

Let $b \in B$. Then $b=t x(\overline{t x})^{-1}$ for some $t \in T, x \in X$. Let us suppose that $t=x_{1} \cdots x_{k-1}$ and $(\overline{t x})^{-1}=x_{k+1} \cdots x_{n}$ in reduced form. We put $x_{k}=x$ so that $b=x_{1} \cdots x_{n}$, although this product may not be reduced as written. Set $t_{i}=\overline{x_{1} \cdots x_{i}}$, for $i=0, \ldots, n$. Using that Schreier transversals are prefixclosed one easily deduces the formulas:

$$
\begin{array}{ll}
t_{i}=x_{1} \cdots x_{i} & \text { for } i<k, \\
t_{i}=x_{n}^{-1} \cdots x_{i+1}^{-1} & \text { for } i>k .
\end{array}
$$


Indeed, the first formula is clear. The second follows because, for $i \geq k+1$, $H t_{i}=H t x x_{k+1} \cdots x_{i}=H x_{n}^{-1} \cdots x_{k+1}^{-1} x_{k+1} \cdots x_{i}=H x_{n}^{-1} \cdots x_{i+1}^{-1}$.

Our aim now is to verify $\pi_{G} \tau(b)=\alpha(b)$. Put $\tau(r)=\left(f_{r}^{\prime}, \rho(r)\right)$, for $r \in F$. We claim that if $t_{H w} x t_{H w x}^{-1}=1\left(x \in X \cup X^{-1}, w \in F\right)$, then $f_{x}^{\prime}(H w)=1$. This is immediate if $x \in X$, since $f_{x}^{\prime}=\alpha f_{x}$ and $f_{x}(H w)=t_{H w} x t_{H w x}^{-1}$. Next assume $x \in X^{-1}$. Hence, taking into account that $x^{-1} \in X$,

$$
f_{x}^{\prime}(H w)=\left(\alpha f_{x^{-1}}(H w x)\right)^{-1}=\left(\alpha\left(t_{H w x} x^{-1} t_{H w}^{-1}\right)\right)^{-1}=1,
$$

since $t_{H w x} x^{-1} t_{H w}^{-1}=\left(t_{H w} x t_{H w x}^{-1}\right)^{-1}=1$.

In light of (3.2) it follows that $t_{i-1} x_{i} t_{i}^{-1}=1$ for all $i \neq k$. Thus by the claim and Lemma 2.3.2,

$$
\begin{aligned}
\pi_{G} \tau(b) & =f_{b}^{\prime}(H)=f_{x_{1} \cdots x_{n}}^{\prime}(H) \\
& =f_{x_{1}}^{\prime}(H) f_{x_{2}}^{\prime}\left(H x_{1}\right) \cdots f_{x_{n}}^{\prime}\left(H x_{1} \cdots x_{n-1}\right) \\
& =f_{x_{1}}^{\prime}\left(H t_{0}\right) f_{x_{2}}^{\prime}\left(H t_{1}\right) \cdots f_{x_{n}}^{\prime}\left(H t_{n-1}\right) \\
& =f_{x_{k}}^{\prime}\left(H t_{k-1}\right)=\alpha\left(t_{k-1} x_{k} t_{k}^{-1}\right)=\alpha(b)
\end{aligned}
$$

as required. This completes the proof that $H$ is freely generated by $B$.

REMARK 3.2.2. Notice that the above proof only shows that $B$ is a basis for $H$. It does not follow from the proof that $B$ is in bijection with the set of pairs $(t, x) \in T \times X$ such that $t x(\overline{t x})^{-1} \neq 1$, although this can be deduced by straightforward combinatorial reasoning.

\subsection{THE NIELSEN-SCHREIER THEOREM FOR FREE PROFINITE GROUPS}

Let $X$ be a profinite space (i.e., a compact Hausdorff and totally disconnected topological space). Then a pro- $\mathcal{C}$ group $F$ is said to be a free pro- $\mathcal{C}$ group on $X$ if there is a continuous map $\iota: X \rightarrow F$ such that if $\sigma: X \rightarrow G$ is any continuous map into a pro- $\mathcal{C}$ group $G$, then there is a unique continuous homomorphism $\psi: F \rightarrow G$ such that

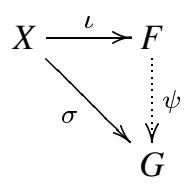

commutes. If $(X, *)$ is a pointed profinite space, one defines in an analogous manner the concept of free pro- $\mathcal{C}$ group on $(X, *)$ : it satisfies the same universal property as above, but with all the maps assumed to be continuous 
maps of pointed spaces (the maps send distinguished points to distinguished points; the distinguished point of a group being its identity element). The map $\iota$ is an embedding, and we identify $X$ with its image $\iota(X)$.

Observe that a free pro- $\mathcal{C}$ group $F$ on a profinite space $X$ can be viewed as free pro- $\mathcal{C}$ group on the pointed profinite space $(X \sqcup\{*\}, *)$ in an obvious way; so we deal here only with pointed spaces. Let $\Phi$ denote the abstract subgroup of $F$ generated by $X$. Then (cf. [18, Propositions 3.3.13 and 3.3.15]), $\Phi$ is a free abstract group with basis $X-\{*\}$; furthermore $\Phi$ is dense in $F$.

Let $H$ be an open subgroup of $F$. Then the natural map

$$
(H \cap \Phi) \backslash \Phi \rightarrow H \backslash F=\Sigma
$$

is a bijection. Choose a Schreier transversal $T$ of $H \cap \Phi$ in $\Phi$. The map

$$
T \times X \rightarrow B=\left\{\operatorname{tx}(\overline{t x})^{-1} \mid t \in T, x \in X\right\} \subseteq H \leq F
$$

given by $(t, x) \mapsto t x(\overline{t x})^{-1}=t x\left(t_{\pi(t x)}\right)^{-1}$ (where $\pi: F \rightarrow \Sigma=H \backslash F$ is the projection) is continuous, since $\pi$ and the section $H f \mapsto t_{H f}$ from $H \backslash F$ to $F$ are obviously continuous. And so, $B$ is closed by the compactness of $T \times X$, i.e., $B$ is profinite. Observe that $1 \in B$. We think of $B$ as a pointed space with distinguished point 1 . The proof of the Nielsen-Schreier Theorem that we have presented above now goes through mutatis mutandis to show that $H$ is a free profinite group on the pointed space $(B, 1)$. Thus we have:

THEOREM 3.3.1. Let $\mathcal{C}$ be an extension closed variety of finite groups. Let $F$ be a free pro- $\mathrm{C}$ group on a pointed profinite space $(X, *)$ and let $H$ be an open subgroup of $F$. Then $H$ is a free pro- $\mathcal{C}$ group on a pointed profinite space.

\section{THE Kurosh THEOREM}

In this section, we give what may arguably be considered the first algebraic proof of the Kurosh Theorem on subgroups of free products. The original proof is essentially combinatorial, while modern proofs have a topological character. Perhaps, Higgin's proof can also be considered algebraic, but it relies on groupoids [10]. Our proof has a similar flavor to the above proof of the Nielsen-Schreier Theorem in that it relies on wreath products. A key difference is that the transversals used are more complicated. 


\subsection{FREE PRODUCTS}

Let $G=*_{\alpha \in A} G_{\alpha}$ be the free product of the groups $G_{\alpha}, \alpha \in A$. We shall freely use the Normal Form Theorem for free products [13, Chap.IV], stating that each non-trivial element $g$ of $G$ can be uniquely written in the form $g=g_{1} g_{2} \cdots g_{m}$ where each $g_{i}$ belongs to some $G_{\alpha}$ and $g_{i} \in G_{\alpha}$ implies $g_{i+1} \notin G_{\alpha}$, for $i=1, \ldots, m-1$. The number $m$ will be called the syllable length of $g$ and we write $\ell(g)=m$. If $S \subseteq G$, denote by $\ell(S)$ the smallest syllable length of an element of $S$. By convention, the syllable length of the identity is 0 . If $g_{m} \in G_{\alpha}$, then we shall say that $g$ ends in the syllable $\alpha$ or that $\alpha$ is the last syllable of $g$.

\subsection{KUROSH SYSTEMS}

Let us begin by setting up notation. Suppose that $G=*_{\alpha \in A} G_{\alpha}$. Let $H \leq G$ and set $\Sigma=H \backslash G$. Denote by $\rho: G \rightarrow S_{\Sigma}$ the associated permutation representation. Let $\left\{H_{i} \mid i \in I\right\}$ be the right cosets of $H$ and assume there is a symbol $1 \in I$ such that $H_{1}=H$. Assume that we have a transversal $T_{\alpha}$ of the right cosets of $H$ in $G$ for each $\alpha \in A$. Denote by $\alpha\left(H_{i}\right)$ the representative of $H_{i}$ in $T_{\alpha}$. We require $\alpha(H)=1$, all $\alpha \in A$.

Definition 4.2.1 (Kurosh system). A collection $D=\left\{D_{\alpha} \mid \alpha \in A\right\}$ of systems $D_{\alpha}$ of representatives $\alpha\left(H g G_{\alpha}\right)$ of the double cosets $H \backslash G / G_{\alpha}$, $\alpha \in A$, together with a system $\left\{T_{\alpha} \mid \alpha \in A\right\}$ of transversals for $H \backslash G$ is called a Kurosh system if the following holds:

(i) if $g=\alpha\left(H g G_{\alpha}\right)$, then $g=\alpha(H g)$;

(ii) $\alpha\left(H g G_{\alpha}\right)$ is either 1 or ends in a syllable $\beta \neq \alpha$;

(iii) $H_{i} \subseteq H g G_{\alpha}$ and $\alpha\left(H g G_{\alpha}\right)=g$ implies $\alpha\left(H_{i}\right) \in g G_{\alpha}$;

(iv) if $1 \neq g=\alpha\left(H g G_{\alpha}\right)$ ends in the syllable $\beta$, then $\beta(H g)=g$;

(v) $\ell\left(\alpha\left(H g G_{\alpha}\right)\right)=\ell\left(H g G_{\alpha}\right)$.

Proposition 4.2.2. Kurosh systems exist.

Proof. We proceed by induction on the length of the double cosets $H g G_{\alpha}$. If $\ell\left(H g G_{\alpha}\right)=0$, i.e. $H g G_{\alpha}=H G_{\alpha}$, choose $\alpha\left(H g G_{\alpha}\right)=1$ and $\alpha(H)=1$; if $H \neq H_{i} \subseteq H G_{\alpha}$, choose $a_{\alpha} \in G_{\alpha}$ so that $H_{i}=H a_{\alpha}$, and put $\alpha\left(H_{i}\right)=a_{\alpha}$. Then conditions (i)-(v) hold. Let $n>1$, and assume representatives $\beta\left(H r G_{\beta}\right)$ and $\beta\left(H_{i}\right)$ have been chosen whenever $H_{i} \subseteq H r G_{\beta}$ and $\ell\left(H r G_{\beta}\right) \leq n-1$ $(\beta \in A, r \in G)$, satisfying conditions (i)-(v). Let $\ell\left(H g G_{\alpha}\right)=n$ with $\ell(g)=n$. Then $g=\bar{g} a_{\beta}$, where $\ell(\bar{g})=n-1,1 \neq a_{\beta} \in G_{\beta}$ and $\beta \neq \alpha$. Since 
$\ell\left(H g G_{\beta}\right) \leq n-1$, representatives $\beta\left(H g G_{\beta}\right)=t$ and $\beta(H g)=t b_{\beta}\left(b_{\beta} \in G_{\beta}\right)$ have already been chosen; in particular, $\ell(t) \leq n-1$ by $(\mathrm{v})$. Since $\ell(H g)=n$, we deduce that $b_{\beta} \neq 1$ and $\ell\left(t b_{\beta}\right)=n$. Define $\alpha\left(H g G_{\alpha}\right)=t b_{\beta}=\alpha(H g)$, and whenever $H g \neq H_{i} \subseteq H g G_{\alpha}$, choose $c_{\alpha} \in G_{\alpha}$ so that $H_{i}=H g c_{\alpha}$, and put $\alpha\left(H_{i}\right)=t b_{\beta} c_{\alpha}$. Clearly, conditions (i)-(v) are satisfied.

Let us define some key elements of $H$. Fix an index $\alpha_{0} \in A$. For $x \in G_{\alpha}$ and $H_{i} \in H \backslash G$, define :

$$
\begin{aligned}
y_{i, x} & =\alpha\left(H_{i}\right) x \alpha\left(H_{i} x\right)^{-1} ; \\
z_{i, \alpha} & =\alpha\left(H_{i}\right) \alpha_{0}\left(H_{i}\right)^{-1} .
\end{aligned}
$$

It is immediate that $y_{i, x}, z_{i, \alpha} \in H$ for all $i, x$ and $\alpha$. Notice that $z_{1, \alpha}=1=z_{i, \alpha_{0}}$ for all $\alpha \in A, i \in I$. If $H_{i}=H g$, we often write $y_{H g, x}$ and $z_{H g, \alpha}$ for $y_{i, x}$ and $z_{i, \alpha}$. We begin with some simple observations concerning these elements.

PROPOSITION 4.2.3. Retaining the above notation, we have:

(1) if $x_{1}, x_{2} \in G_{\alpha}$, then $y_{i, x_{1}} y_{j, x_{2}}=y_{i, x_{1} x_{2}}$ where $H_{i} x_{1}=H_{j}$;

(2) if $x \in G_{\alpha}, H_{i} \subseteq H u G_{\alpha}$ with $u=\alpha\left(H u G_{\alpha}\right)$, then $y_{i, x} \in u G_{\alpha} u^{-1} \cap H$;

(3) if $h \in u G_{\alpha} u^{-1} \cap H$ with $u=\alpha\left(H u G_{\alpha}\right)$, then $h=y_{H u, x}$ for some $x \in G_{\alpha}$;

(4) if $1 \neq u=\alpha\left(H u G_{\alpha}\right)$ ends with the syllable $\beta$, then $z_{H u, \alpha}=z_{H u, \beta}$.

Proof. First we handle (1). The definition yields

$$
y_{i, x_{1}} y_{j, x_{2}}=\alpha\left(H_{i}\right) x_{1} \alpha\left(H_{i} x_{1}\right)^{-1} \alpha\left(H_{i} x_{1}\right) x_{2} \alpha\left(H_{i} x_{1} x_{2}\right)^{-1}=y_{i, x_{1} x_{2}} .
$$

Next we turn to (2). By condition (iii) of a Kurosh system, $\alpha\left(H_{i}\right)=u g$ and $\alpha\left(H_{i} x\right)=u g^{\prime}$ for some $g, g^{\prime} \in G_{\alpha}$, whence $y_{i, x}=u g x\left(u g^{\prime}\right)^{-1} \in u G_{\alpha} u^{-1} \cap H$. To prove (3), suppose $h=u x u^{-1}$ with $x \in G_{\alpha}$. Then $H u=H u x$ and $\alpha(H u)=u$ by (i). We conclude that $y_{H u, x}=\alpha(H u) x \alpha(H u x)^{-1}=u x u^{-1}=h$. For (4) we simply observe that $\alpha(H u)=u=\beta(H u)$ by (i) and (iv).

\subsection{The Kurosh TheOrem}

Set $Z=\left\{z_{i, \alpha} \mid i \in I, \alpha \in A, z_{i, \alpha} \neq 1\right\}$ and $F=\langle Z\rangle$. Our goal is to prove

$$
\left.H=\underset{\alpha \in A}{*} \underset{u \in D_{\alpha}}{*}\left(u G_{\alpha} u^{-1} \cap H\right)\right] * F
$$

and $F$ is freely generated by $Z$. We use wreath products and the universal property to effect this proof. From now on we work with a fixed Kurosh system. If $\psi: Z \rightarrow K$ is a map, with $K$ a group, then we extend $\psi$ to $Z \cup\{1\}$ by setting $\psi(1)=1$. 
Proposition 4.3.1. Given a family $\mathcal{F}=\left\{\psi_{u}: u G_{\alpha} u^{-1} \cap H \rightarrow K\right\}_{\alpha \in A, u \in D_{\alpha}}$ of group homomorphisms and a map $\psi: Z \rightarrow K$, there exists, for each $\alpha \in A$, a homomorphism $\Psi_{\alpha}: G_{\alpha} \rightarrow K \curlywedge \rho(G)$ defined by $\Psi_{\alpha}(x)=\left(f_{x}, \rho(x)\right)$ with

$$
f_{x}\left(H_{i}\right)=\psi\left(z_{i, \alpha}\right)^{-1} \psi_{u}\left(y_{i, x}\right) \psi\left(z_{j, \alpha}\right),
$$

where $H_{i} x=H_{j}$ and $u=\alpha\left(H_{i} G_{\alpha}\right)$. If $\Psi: G \rightarrow K \imath \rho(G)$ denotes the induced homomorphism, then the following diagram commutes:

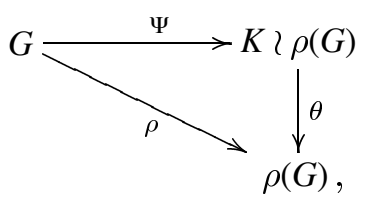

where $\theta$ is the projection.

Moreover, the construction of $\Psi$ is functorial in the sense that given another family of homomorphisms $\mathcal{F}^{\prime}=\left\{\psi_{u}^{\prime}: u G_{\alpha} u^{-1} \cap H \rightarrow K^{\prime}\right\}_{\alpha \in A, u \in D_{\alpha}}$, a map $\psi^{\prime}: Z \rightarrow K^{\prime}$ and a homomorphism $\gamma: K^{\prime} \rightarrow K$ such that the diagrams
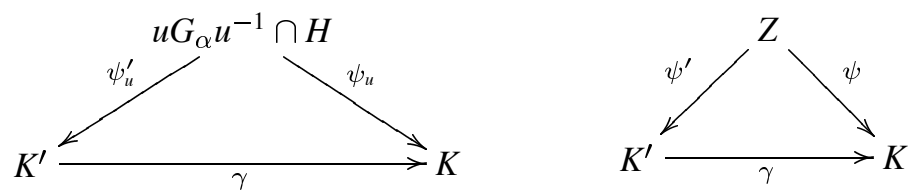

commute, then the following diagram commutes:

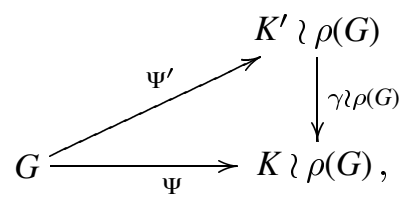

where $\Psi^{\prime}$ is the map associated to the family $\mathcal{F}^{\prime}$.

Proof. We begin by verifying that $\Psi_{\alpha}$ is a homomorphism. Proposition 4.2.3(2) implies that $y_{i, x} \in u G_{\alpha} u^{-1} \cap H$ so that $\Psi_{\alpha}$ makes sense. Let $x_{1}, x_{2} \in G_{\alpha}$. Clearly, $H_{i} x_{1} G_{\alpha}=H_{i} x_{2} G_{\alpha}=H_{i} x_{1} x_{2} G_{\alpha}=H_{i} G_{\alpha}$; set $u=\alpha\left(H_{i} G_{\alpha}\right)$. From

$$
\left(f_{x_{1}}, \rho\left(x_{1}\right)\right)\left(f_{x_{2}}, \rho\left(x_{2}\right)\right)=\left(f_{x_{1}}\left({ }^{\rho\left(x_{1}\right)} f_{x_{2}}\right), \rho\left(x_{1} x_{2}\right)\right)
$$

it follows that we just need $f_{x_{1}}\left(H_{i}\right) f_{x_{2}}\left(H_{i} x_{1}\right)=f_{x_{1} x_{2}}\left(H_{i}\right)$. Putting $H_{j}=H_{i} x_{1}$ 
and $H_{k}=H_{i} x_{1} x_{2}$, an application of Proposition 4.2.3(1) yields

$$
\begin{aligned}
f_{x_{1}}\left(H_{i}\right) f_{x_{2}}\left(H_{i} x_{1}\right) & =\psi\left(z_{i, \alpha}\right)^{-1} \psi_{u}\left(y_{i, x_{1}}\right) \psi\left(z_{j, \alpha}\right) \psi\left(z_{j, \alpha}\right)^{-1} \psi_{u}\left(y_{j, x_{2}}\right) \psi\left(z_{k, \alpha}\right) \\
& =\psi\left(z_{i, \alpha}\right)^{-1} \psi_{u}\left(y_{i, x_{1} x_{2}}\right) \psi\left(z_{k, \alpha}\right)=f_{x_{1} x_{2}}\left(H_{i}\right)
\end{aligned}
$$

as required. The $\Psi_{\alpha}$ induce the desired map $\Psi$ by the universal property of a free product. The commutativity of (4.1) and (4.3) are immediate from the definition of $\Psi_{\alpha}$ and the universal property of a free product.

From the proposition and Lemma 2.3.4, we obtain

Corollary 4.3.2. Let $\Psi, \Psi^{\prime}$ and $\gamma$ be as in Proposition 4.3.1. Then there is a commutative diagram

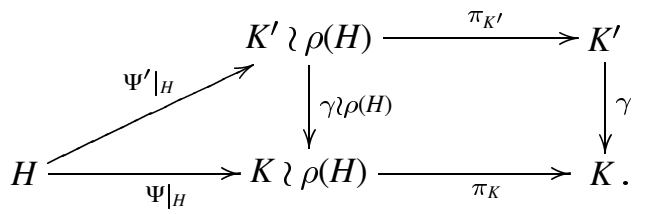

Our next lemma is where we make use of the full strength of the Kurosh system.

LEMMA 4.3.3. Let $u=\alpha(H u)$. Then $f_{u}(H)=\psi\left(z_{H u, \alpha}\right)$.

Proof. We induct on the syllable length of $u$. If $u=1$, there is nothing to prove as $z_{H, \alpha}=1$ for all $\alpha$. So assume $u \neq 1$. The proof divides into two cases.

CASE 1. Assume $u \neq \alpha\left(H u G_{\alpha}\right)$. Then (iii) implies that we can write $u=v x$ with $v=\alpha\left(H u G_{\alpha}\right)$ and $x \in G_{\alpha}$. Moreover, $\ell(v)<\ell(u)$ by (ii). Since $\alpha(H v)=v$ by (i), by induction $f_{v}(H)=\psi\left(z_{H v, \alpha}\right)$. Then we find by Lemma 2.3.2

$$
\begin{aligned}
f_{u}(H)=f_{v}(H) f_{x}(H v) & =\psi\left(z_{H v, \alpha}\right) \psi\left(z_{H v, \alpha}\right)^{-1} \psi_{v}\left(y_{H v, x}\right) \psi\left(z_{H u, \alpha}\right) \\
& =\psi_{v}\left(y_{H v, x}\right) \psi\left(z_{H u, \alpha}\right) .
\end{aligned}
$$

But $y_{H v, x}=\alpha(H v) x \alpha(H v x)^{-1}=\alpha(H v) x \alpha(H u)^{-1}=v x u^{-1}=1$, establishing $f_{u}(H)=\psi\left(z_{H u, \alpha}\right)$. 
CASE 2. Suppose $u=\alpha\left(H u G_{\alpha}\right)$. Since $u \neq 1$, (ii) implies that $u$ ends in a syllable $\beta$ with $\beta \neq \alpha$ and (iv) yields $\beta(H u)=u$. By (ii) $u \neq \beta\left(H u G_{\beta}\right)$, so Case 1 implies that $f_{u}(H)=\psi\left(z_{H u, \beta}\right)$. Proposition 4.2.3 (4) provides $z_{H u, \beta}=z_{H u, \alpha}$, so $f_{u}(H)=\psi\left(z_{H u, \alpha}\right)$.

This establishes the lemma.

An important special case is when $K=H$ and the $\psi_{u}$ and $\psi$ are the inclusions. Let us denote the induced map in this case by $\widetilde{\Psi}: G \rightarrow H \imath \rho(G)$.

PROPOSITION 4.3.4. The map $\widetilde{\Psi}: G \rightarrow H<\rho(G)$ is the standard wreath product embedding associated to the transversal $T_{\alpha_{0}}$. Consequently, $\left.\pi_{H} \widetilde{\Psi}\right|_{H}$ is the identity.

Proof. Writing $\widetilde{\Psi}(g)=\left(F_{g}, \rho(g)\right)$, if $x \in G_{\alpha}$ and $H_{i} x=H_{j}$, then

$$
\begin{aligned}
F_{x}\left(H_{i}\right)=z_{i, \alpha}^{-1} y_{i, x} z_{j, \alpha} & =\alpha_{0}\left(H_{i}\right) \alpha\left(H_{i}\right)^{-1}\left[\alpha\left(H_{i}\right) x \alpha\left(H_{j}\right)^{-1}\right] \alpha\left(H_{j}\right) \alpha_{0}\left(H_{j}\right)^{-1} \\
& =\alpha_{0}\left(H_{i}\right) x \alpha_{0}\left(H_{i} x\right)^{-1} .
\end{aligned}
$$

Thus $\widetilde{\Psi}$ is the standard embedding associated to the transversal $T_{\alpha_{0}}$.

In the proof of the next theorem, we retain all the notation introduced in this section.

THEOREM 4.3 .5 (Kurosh). Let $\left\{D_{\alpha}, T_{\alpha} \mid \alpha \in A\right\}$ be a Kurosh system for $H \leq G=*_{\alpha \in A} G_{\alpha}$. Then

$$
H=\underset{\alpha \in A}{*}\left[\underset{u \in D_{\alpha}}{*}\left(u G_{\alpha} u^{-1} \cap H\right)\right] * F
$$

and $F$ is a free group with basis $Z$.

Proof. Let $\left\{\psi_{u}: u G_{\alpha} u^{-1} \cap H \rightarrow K\right\}_{\alpha \in A, u \in D_{\alpha}}$ be a family of group homomorphisms and $\psi: Z \rightarrow K$ a map. Let $\Psi: G \rightarrow K 2 \rho(G)$ be as in Proposition 4.3.1. We show that $\left.\pi_{K} \Psi\right|_{H}$ extends the $\psi_{u}$ and $\psi$, where $\pi_{K}=\pi_{K, 1}$ is as in Lemma 2.3.4. Suppose $u=\alpha\left(H u G_{\alpha}\right)$ and $h \in u G_{\alpha} u^{-1} \cap H$. By Proposition 4.2.3(3), $h=y_{i, x}$ for some $x \in G_{\alpha}$, where $H_{i}=H u$. Setting $H_{j}=H_{i} x$, an application of Lemmas 4.3.3 and 2.3.2 (and the fact 
$\left.H \alpha\left(H_{i}\right)=H_{i}\right)$ yields

$$
\begin{aligned}
\pi_{K} \Psi(h) & =f_{y_{i, x}}(H)=f_{\alpha\left(H_{i}\right) x \alpha\left(H_{j}\right)^{-1}}(H) \\
& =f_{\alpha\left(H_{i}\right)}(H) f_{x}\left(H_{i}\right)\left(f_{\alpha\left(H_{j}\right)}\left(H_{j} \alpha\left(H_{j}\right)^{-1}\right)\right)^{-1} \\
& =f_{\alpha\left(H_{i}\right)}(H) f_{x}\left(H_{i}\right)\left(f_{\alpha\left(H_{j}\right)}(H)\right)^{-1} \\
& =\psi\left(z_{i, \alpha}\right)\left[\psi\left(z_{i, \alpha}\right)^{-1} \psi_{u}\left(y_{i, x}\right) \psi\left(z_{j, \alpha}\right)\right] \psi\left(z_{j, \alpha}\right)^{-1} \\
& =\psi_{u}\left(y_{i, x}\right)=\psi_{u}(h)
\end{aligned}
$$

Similarly, we calculate using Lemmas 4.3.3 and 2.3.2

$$
\begin{aligned}
\pi_{K} \Psi\left(z_{i, \alpha}\right) & =f_{z_{i, \alpha}}(H)=f_{\alpha\left(H_{i}\right)}(H)\left(f_{\alpha_{0}\left(H_{i}\right)}\left(H_{i} \alpha_{0}\left(H_{i}\right)^{-1}\right)\right)^{-1} \\
& =f_{\alpha\left(H_{i}\right)}(H)\left(f_{\alpha_{0}\left(H_{i}\right)}(H)\right)^{-1} \\
& =\psi\left(z_{i, \alpha}\right) \psi\left(z_{i, \alpha_{0}}\right)^{-1}=\psi\left(z_{i, \alpha}\right)
\end{aligned}
$$

since $z_{i, \alpha_{0}}=1$.

The uniqueness of $\left.\pi_{K} \Psi\right|_{H}$ follows from the functoriality of our construction. Namely, in Proposition 4.3.1 take $K^{\prime}=H$ and $\psi_{u}^{\prime}, \psi^{\prime}$ the inclusions (and so $\Psi^{\prime}: G \rightarrow H \gamma \rho(G)$ is $\widetilde{\Psi}$ from Proposition 4.3.4). Suppose $\gamma: H \rightarrow K$ is an extension of the $\psi_{u}$ and $\psi$. Then (4.2) commutes and so diagrams (4.3) and (4.4) commute. Since $\left.\pi_{H} \Psi^{\prime}\right|_{H}=\left.\pi_{H} \widetilde{\Psi}\right|_{H}$ is the identity in this case by Proposition 4.3.4, we conclude that $\gamma=\left.\pi_{K} \Psi\right|_{H}$.

REMARK 4.3.6. As we mentioned earlier, there is a close relationship between the standard embedding and induced representations [21]. From this viewpoint, our proof of the Kurosh Theorem has a similar flavor to Mackey's Theorem on the restriction to one subgroup of a representation induced from another.

\subsection{The Kurosh Subgroup THEOREM FOR PROFINITE GROUPS}

Let $\Gamma$ be a pro- $\bigodot$ group and let $\left\{\Gamma_{\alpha} \mid \alpha \in A\right\}$ be a collection of pro- $\bigodot$ groups indexed by a set $A$. For each $\alpha \in A$, let $\iota_{\alpha}: \Gamma_{\alpha} \rightarrow \Gamma$ be a continuous homomorphism. One says that the family $\left\{\iota_{\alpha} \mid \alpha \in A\right\}$ is convergent if whenever $U$ is an open neighborhood of 1 in $\Gamma$, then $U$ contains all but a finite number of the images $\iota_{\alpha}\left(\Gamma_{\alpha}\right)$. We say that $\Gamma$ together with the $\iota_{\alpha}$ is the free pro- $\bigodot$ product of the groups $\Gamma_{\alpha}$ if the following universal property is satisfied: whenever $K$ is a pro- $\bigodot$ group and $\left\{\lambda_{\alpha}: \Gamma_{\alpha} \rightarrow K \mid \alpha \in A\right\}$ is a convergent family of continuous homomorphisms, then there exists a unique 
continuous homomorphism $\lambda: \Gamma \rightarrow K$ such that

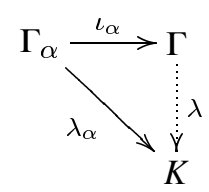

commutes, for all $\alpha \in A$. We denote such a free pro- $\mathcal{C}$ product by $\Gamma=\coprod_{\alpha \in A} \Gamma_{\alpha}$. Free pro- $\mathcal{C}$ products exist and are unique. To construct the free pro- $\mathcal{C}$ product $\Gamma$ one proceeds as follows: let $G=*_{\alpha \in A} \Gamma_{\alpha}$ be the free product of the groups $\Gamma_{\alpha}$ as abstract groups. Consider the pro- $\mathcal{C}$ topology on $G$ determined by the collection of normal subgroups $N$ of finite index in $G$ such that $G / N \in \mathcal{C}, N \cap \Gamma_{\alpha}$ is open in $\Gamma_{\alpha}$, for each $\alpha \in A$, and $N \geq \Gamma_{\alpha}$, for all but finitely many $\alpha$. Then

$$
\Gamma=\lim _{\stackrel{N}{ }} G / N .
$$

It turns out that $G$ is naturally embedded in $\Gamma$ as a dense subgroup. One can take the homomorphism $\iota_{\alpha}$ to be the composition of inclusions

$$
\Gamma_{\alpha} \hookrightarrow G \hookrightarrow \Gamma \quad(\alpha \in A) .
$$

If the set $A$ is finite, the 'convergence' property of the homomorphisms $\iota_{\alpha}$ is automatic.

For such free products, one has the following analogue of the Kurosh Subgroup Theorem [3].

THEOREM 4.4.1. Let $H$ be an open subgroup of the free pro- $\mathrm{C}$ product

$$
\Gamma=\coprod_{\alpha \in A} \Gamma_{\alpha} .
$$

Then, for each $\alpha \in A$, there exists a set $D_{\alpha}$ of representatives of the double cosets $H \backslash \Gamma / \Gamma_{\alpha}$ such that the family of inclusions

$$
\left\{u \Gamma_{\alpha} u^{-1} \cap H \hookrightarrow H \mid u \in D_{\alpha}, \alpha \in A\right\}
$$

converges, and $\mathrm{H}$ is the free pro- $\mathrm{C}$ product

$$
H=\left[\coprod_{\alpha \in A, u \in D_{\alpha}} u \Gamma_{\alpha} u^{-1} \cap H\right] \amalg \Phi,
$$

where $\Phi$ is a free pro- $\mathcal{C}$ group of finite rank. 
Proof. First we show that we may assume that $A$ is finite. Consider the core $H_{\Gamma}=\bigcap_{g \in \Gamma} g H g^{-1}$ of $H$ in $\Gamma$. Since $H$ is open, we have that $H_{\Gamma}$ is open in $\Gamma$. So there exists a finite subset $B$ of $A$ such that $\Gamma_{\alpha} \leq H_{\Gamma}$ for all $\alpha \in A-B$. Put $\Gamma^{\prime}=\coprod_{\alpha \in A-B} \Gamma_{\alpha}$; then

$$
\Gamma=\left[\coprod_{\alpha \in B} \Gamma_{\alpha}\right] \amalg \Gamma^{\prime}
$$

is a free pro- $\mathcal{C}$ product of finitely many factors, and one easily sees that it suffices to prove the theorem for this product. Indeed, observe first that for all $\alpha \in A-B, H_{\Gamma} \geq \Gamma_{\alpha}$ and since $H_{\Gamma} \triangleleft \Gamma$, one has $H u \Gamma_{\alpha}=H u=H u \Gamma^{\prime}$ $(u \in \Gamma)$, i.e., $H \backslash \Gamma / \Gamma^{\prime}=H \backslash \Gamma=H \backslash \Gamma / \Gamma_{\alpha}$; on the other hand,

$$
u \Gamma^{\prime} u^{-1} \cap H=u \Gamma^{\prime} u^{-1}=\coprod_{\alpha \in A-B} u \Gamma_{\alpha} u^{-1}=\coprod_{\alpha \in A-B}\left(u \Gamma_{\alpha} u^{-1} \cap H\right) .
$$

Hence from now on we assume that $A$ is a finite indexing set.

Choose a Kurosh system $\left\{D_{\alpha}, T_{\alpha} \mid \alpha \in A\right\}$ for the subgroup $G \cap H$ of the abstract free product $G=*_{\alpha \in A} \Gamma_{\alpha}$, and observe that, for each $\alpha, T_{\alpha}$ and $D_{\alpha}$ are systems of representatives of the cosets $H \backslash G$ and of the double cosets $H \backslash \Gamma / \Gamma_{\alpha}$, respectively. The remainder of the proof can be carried out mutatis mutandis as is done in the proof of Theorem 4.3 .5 (one simply has to require initially that the homomorphisms $\psi_{u}$ are continuous, and then verify that all the maps involved in the proof are also continuous; this is an easy consequence of our comments in 2.4).

Let us point out that this proof is independent of the result for abstract free products (Theorem 4.3.5); it simply follows the same procedure.

We leave open the question of whether or not the same simple procedure works in case one deals with pro- $\bigodot$ products of pro- $\varrho$ groups indexed by a profinite space [22].

\section{QUASIFREE PROFINITE GROUPS}

This section contains a simpler proof of the main result of [17]. A similar approach, using the twisted wreath product, was independently discovered by Bary-Soroker et al. [2]. 


\subsection{QUASIFREE GROUPS}

An epimorphism of groups is termed proper if it is not an isomorphism. Let $\mathcal{C}$ be an extension closed variety of finite groups and let $m$ be an infinite cardinal number. A pro- $\bigodot$ group $G$ is called $m$-quasifree if whenever $A$ and $B$ are groups in $\mathcal{C}, \alpha: A \rightarrow B$ is a proper epimorphism of groups that splits (i.e., there is a section $\sigma: B \rightarrow A$ of $\alpha: \alpha \sigma=\operatorname{id}_{B}$ ), and $\beta: G \rightarrow B$ is a continuous epimorphism,

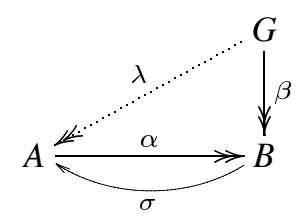

then there exist precisely $m$ different continuous epimorphisms $\lambda: G \rightarrow A$ such that $\alpha \lambda=\beta$. See $[17,9]$ for motivation and elementary properties of these groups; one knows in particular that the minimal number $d(G)$ of generators converging to 1 of such an $m$-quasifree group $G$ is $m$ (see [17, Lemma 1.2]). In [17] it is proved that open subgroups of $m$-quasifree groups are $m$-quasifree. Here we provide a simpler and more natural proof of this result by means of wreath products.

THEOREM 5.1.1. Let $G$ be an m-quasifree pro- $\mathrm{C}$ group, and let $H$ be an open subgroup of $G$. Then $H$ is m-quasifree.

Proof. Given $A, B \in \mathcal{C}$, a proper split epimorphism $\alpha: A \rightarrow B$ and a continuous epimorphism $\beta: H \rightarrow B$, we need to prove the existence of exactly $m$ continuous epimorphisms $\lambda: H \rightarrow A$ such that $\alpha \lambda=\beta$.

Set $\Sigma=H \backslash G$ and let $\rho: G \rightarrow S_{\Sigma}$ be the corresponding permutation representation as in Section 2. Consider the standard embedding

$$
\varphi: G \rightarrow H(\rho(G)
$$

constructed in Theorem 2.3.1. Note that $\alpha \imath \rho(G): A \imath \rho(G) \rightarrow B \imath \rho(G)$ is a split proper epimorphism by Proposition 2.2.1; observe also that $A 2 \rho(G)$ and $B \imath \rho(G)$ are finite groups in $\mathcal{C}$, as $\mathcal{C}$ is extension closed. Let $B^{\prime}=(\beta \imath \rho(G)) \varphi(G)$ and $A^{\prime}=(\alpha<\rho(G))^{-1}\left(B^{\prime}\right)$. Then $A^{\prime}, B^{\prime} \in \mathcal{C}$, and the restriction $\alpha^{\prime}: A^{\prime} \rightarrow B^{\prime}$ of $\alpha 2 \rho(G)$ to $A^{\prime}$ is a split proper epimorphism. See Figure 1. Since $G$ is $m$-quasifree, there exists a continuous epimorphism $\tilde{\lambda}: G \rightarrow A^{\prime}$ such that $\alpha^{\prime} \tilde{\lambda}=(\beta \imath \rho(G)) \varphi$. Then, for each $g \in G, \widetilde{\lambda}(g)=\left(\tilde{f}_{g}, \rho(g)\right)$, for some $\widetilde{f}_{g} \in A^{\Sigma}$. 


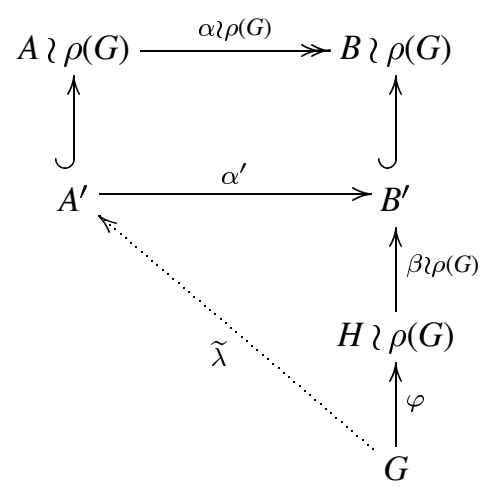

FIGURE 1

A commutative diagram

Let $T=\left\{t_{1}=1, t_{2}, \ldots, t_{k}\right\}$ be a right transversal of $H$ in $G$. For $i=1, \ldots, k$, define $\lambda_{i}: H^{t_{i}} \rightarrow A$ to be $\lambda_{i}=\left.\pi_{A, i} \widetilde{\lambda}\right|_{H^{t_{i}}}$, i.e., $\lambda_{i}(x)=\widetilde{f}_{x}\left(H t_{i}\right)$, for $x \in H^{t_{i}}$. According to Lemma 2.3.4, the diagram

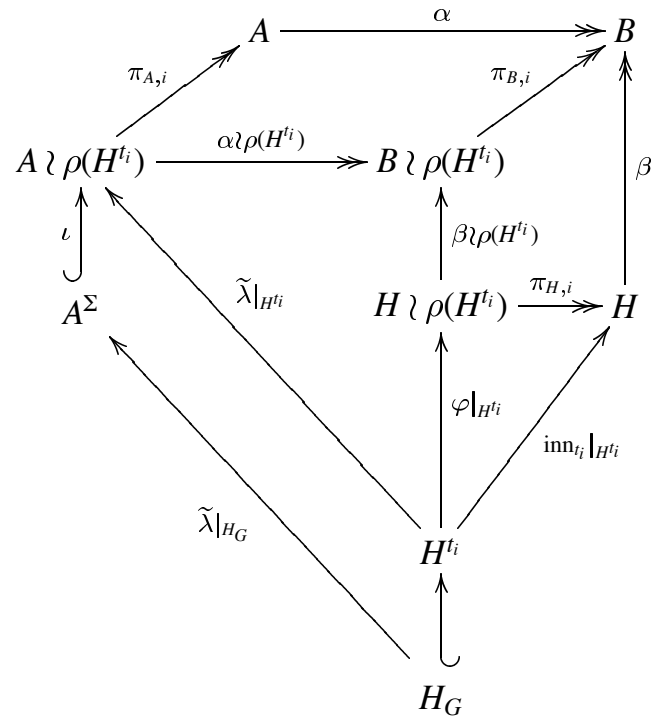

commutes. Thus $\left.\beta \circ \operatorname{inn}_{t_{i}}\right|_{H^{t_{i}}}=\alpha \lambda_{i}$.

We claim that $\lambda_{i}$ is surjective. Let $a \in A$ and let $b=\alpha(a)$. Since $\beta$ is surjective, the commutativity of the above diagram ensures that there exists $(f, \rho(x))) \in\left(B \succ \rho\left(H^{t_{i}}\right)\right) \cap B^{\prime}$, where $x \in H^{t_{i}}$, with $f\left(H t_{i}\right)=b$. Choose 
$f^{\prime}: \Sigma \rightarrow A$ to be any function such that $f^{\prime}\left(H t_{i}\right)=a$ and $\alpha f^{\prime}=f$; then $\left(f^{\prime}, \rho(x)\right) \in A^{\prime}$. Therefore, $\pi_{A, i}$ takes $A^{\prime} \cap\left(A<\rho\left(H^{t_{i}}\right)\right)$ onto $A$. Because $\operatorname{ker} \widetilde{\lambda} \leq \operatorname{ker} \rho=H_{G} \leq H^{t_{i}}$, it follows that $\widetilde{\lambda}(g) \in A<\rho\left(H^{t_{i}}\right)$ implies $g \in H^{t_{i}}$. We deduce that $\left.\widetilde{\lambda}\right|_{H^{t i}}: H^{t_{i}} \rightarrow A^{\prime} \cap\left(A \prec \rho\left(H^{t_{i}}\right)\right)$ is an epimorphism, and hence so is $\lambda_{i}$, proving the claim.

Since $G$ is quasifree, the total number of epimorphisms $\widetilde{\lambda}: G \rightarrow A^{\prime}$ such that $\alpha^{\prime} \tilde{\lambda}=(\beta \imath \rho(G)) \varphi$ is $m$. Since $H_{G}=\bigcap_{i=1}^{k} H^{t_{i}}$ has finite index in $G$, these $\widetilde{\lambda}$ restrict to $m$ different homomorphisms

$$
\left.\widetilde{\lambda}\right|_{H_{G}}: H_{G} \rightarrow A \prec \rho\left(H_{G}\right)=A^{\Sigma} .
$$

Recalling from Lemma 2.3.4 that the $\pi_{A, i}: A^{\Sigma} \rightarrow A(i=1, \ldots, k)$ are the direct product projections, we conclude that $\left.\widetilde{\lambda}\right|_{H_{G}}$ is determined by the maps $\left.\pi_{A, i} \tilde{\lambda}\right|_{H_{G}}=\left.\lambda_{i}\right|_{H_{G}}, i=1, \ldots, k$. It follows that there exists some $j \in\{1, \ldots, k\}$, such that the number of different maps $\left.\lambda_{j}\right|_{H_{G}}$ constructed in this manner is precisely $m$.

For each of these $\lambda_{j}$, define $\lambda=\lambda_{j} \circ$ inn $\left._{t_{j}^{-1}}\right|_{H}$. Then, since $H_{G}$ has finite index in $H$, we have constructed $m$ different epimorphisms $\lambda: H \rightarrow A$ such that $\alpha \lambda=\beta$. Finally, observe that there cannot be more such $\lambda$ 's since the minimal number $d(H)$ of generators of $H$ converging to 1 is $m$ and $A$ is finite. This completes the proof.

It is an open question whether the results of [2] for semifree profinite groups also hold for quasifree groups. 


\section{REFERENCES}

[1] Almeida, J. and B. Steinberg. Rational codes and free profinite monoids. J. London Math. Soc. (2) 79 (2009), 465-477.

[2] BARY-SOROKer, L., D. HARAN and D. HARBATER. Permanence criteria for semi-free profinite groups. Preprint arXiv: 0810.0845 (2008). To appear in Math. Ann.

[3] BinZ, E., J. Neukirch and G.H. Wenzel. A subgroup theorem for free products of pro-finite groups. J. Algebra 19 (1971), 104-109.

[4] Cossey, J., O.H. Kegel and L.G. KovÁcs. Maximal Frattini extensions. Archiv der Math. 35 (1980), 210-217.

[5] EILENBerg, S. Automata, Languages, and Machines. Vol. B. With two chapters ("Depth decomposition theorem" and "Complexity of semigroups and morphisms") by B. Tilson. Pure and Applied Mathematics 59. Academic Press, New York-London, 1976.

[6] ERshov, Yu. L. On a theorem of Kurosh. Algebra i Logika 37 (1998), 381-393, 494; translation in Algebra and Logic 37 (1998), 215-222.

[7] HaLl, M., JR. The Theory of Groups. The Macmillan Co., New York, N.Y., 1959.

[8] Haran, D. On closed subgroups of free products of profinite groups. Proc. London Math. Soc. (3) 55 (1987), 266-298.

[9] Harbater, D. and K.F. Stevenson. Local Galois theory in dimension two. Adv. Math. 198 (2005), 623-653.

[10] Higgins, P.J. Categories and groupoids. Repr. Theory Appl. Categ. 7 (2005), 1-178. Reprint of the 1971 original [Notes on Categories and Groupoids, Van Nostrand Reinhold, London] with a new preface by the author.

[11] Krasner, M. et L. KALOUJNINE. Produit complet des groupes de permutations et problème d'extension de groupes. I. Acta Sci. Math. Szeged 13 (1950), 208-230.

[12] Krohn, K. and J. Rhodes. Algebraic theory of machines. I. Prime decomposition theorem for finite semigroups and machines. Trans. Amer. Math. Soc. 116 (1965), 450-464.

[13] LYNDON, R.C. and P.E. SchupP. Combinatorial Group Theory. Reprint of the 1977 edition. Classics in Mathematics. Springer-Verlag, Berlin, 2001.

[14] MARGOLIS, S., M. SAPIR and P. WEIL. Irreducibility of certain pseudovarieties. Comm. Algebra 26 (1998), 779-792.

[15] RHODES, J. and B. STEINBERG. Closed subgroups of free profinite monoids are projective profinite groups. Bull. London Math. Soc. 40 (2008), 375-383.

[16] Rhodes, J. and B. SteInBERG. The q-Theory of Finite Semigroups. Springer Monographs in Mathematics. Springer, New York, 2009.

[17] Ribes, L., K. STEVENSON and P. ZALESSKĬ. On quasifree profinite groups. Proc. Amer. Math. Soc. 135 (2007), 2669-2676.

[18] RiBes, L. and P. ZALESSKII. Profinite Groups. Ergebnisse der Mathematik und ihrer Grenzgebiete, 3. Folge, 40. $2^{\text {nd }}$ ed. Springer-Verlag, Berlin, 2010.

[19] SCHÜTZENBERGER, M.-P. Sur la représentation monomiale des demi-groupes. C. R. Acad. Sci. Paris 246 (1958), 865-867. 
[20] Steinberg, B. Maximal subgroups of the minimal ideal of a free profinite monoid are free. Israel J. Math. (to appear).

[21] WiEland, H. and B. HuPPERT. Arithmetical and normal structure of finite groups. In: Proc. Sympos. Pure Math. VI, 17-38. Amer. Math. Soc., Providence, R.I., 1962.

[22] ZALESSKII, P. A. Open subgroups of free profinite products. In: Proceedings of the International Conference on Algebra, Part 1 (Novosibirsk, 1989), 473-491. Contemp. Math. 131. Amer. Math. Soc., Providence, RI, 1992.

(Reçu le ler décembre 2008)

L. Ribes

B. Steinberg

School of Mathematics and Statistics

Carleton University

1125 Colonel By Drive

Ottawa, Ontario K1S 5B6

Canada

e-mail : lribes@math.carleton.ca, bsteinbg@math.carleton.ca 